\title{
MERCADO PARANAENSE DE MADEIRA EM TORA PROCEDENTE DE SILVICULTURA ENTRE 1999 E 2005
}

\author{
Alexandre Nascimento de Almeida ${ }^{1}$, João Carlos Garzel Leodoro da Silva ${ }^{2}$, Humberto Ângelo ${ }^{3}$, \\ Alexandre Muzy Bittencourt ${ }^{1}$, Blas Enrique Caballero Nuñez ${ }^{4}$ \\ ${ }^{1}$ Eng. Florestal, Doutorando em Ciências Florestais, UFPR, Curitiba, PR, Brasil - alexfloresta@ pop.com.br - alexbitten@terra.com.br \\ ${ }^{2}$ Eng. Florestal, Dr., Depto. de Economia Rural e Extensão, UFPR, Curitiba, PR, Brasil - garzel@ufpr.br \\ ${ }^{3}$ Eng. Florestal, Dr., Depto. de Engenharia Florestal, UnB, Brasília, DF, Brasil - humb@unb.br \\ ${ }^{4}$ Economista, Dr., Depto. de Economia, UFPR, Curitiba, PR, Brasil - blas@ufpr.br \\ Recebido para publicação: 07/08/2008 - Aceito para publicação: 30/04/2009
}

\begin{abstract}
Resumo
Este trabalho analisa o mercado paranaense de madeira em tora no período de 1999 a 2005 . O objetivo foi analisar se o aumento de preço da madeira em tora no período em questão foi provocado por uma variação da demanda ou da oferta de madeira. Foram calculadas as taxas anuais de crescimento do preço e da quantidade de madeira em tora, e estas relacionadas conforme os deslocamentos das curvas de oferta e demanda. Os resultados indicaram que o aumento do preço da madeira em tora no período de 1999 a 2005 foi devido a fatores relacionados, principalmente, à demanda externa.
\end{abstract}

Palavras-chave: Mercado; madeira em tora; Paraná; oferta; demanda.

\begin{abstract}
Analyzis of the Paraná roundwood market from silviculture between 1999 and 2005. This work analyzes the round wood market of Paraná in the period from 1999 to 2005. The objective was to analyze if the increase of price of round wood in the last years was provoked by a variation of the wood demand or wood supply. The annual price increasing and quantity growth taxes of round wood were calculated and related according to the displacements of the supply and demand curves. The results suggested that the round wood price increasing in the period from 1999 to 2005 was due to factors related mainly to the external demand.

Keywords: Market; round wood; Parana; supply; demand.
\end{abstract}

\section{INTRODUÇÃO}

A importância dos produtos florestais na formação do valor bruto da produção agropecuária paranaense (VBP) vem se ampliando nos últimos anos. Os produtos florestais, em 1997, representavam 6,73\% do VBP estadual, enquanto que, em 2004, essa participação foi ampliada para 9,28\% (ANDRETTA, 2006).

De acordo com a Associação Brasileira da Indústria de Madeira Processada Mecanicamente (ABIMCI, 2007), em 2004 o Paraná contou com 553 estabelecimentos de exploração florestal, correspondendo a $14,3 \%$ do total brasileiro. Em relação a estabelecimentos responsáveis pelo desdobramento e fabricação de produtos de madeira, o total contabilizado no Paraná e suas participações no Brasil foram, respectivamente, $1200(17,1 \%)$ e 1294 (14,7\%) estabelecimentos.

Essa mesma Associação mostra, também, a importância na geração de empregos, sendo que a atividade florestal paranaense respondeu por $9,8 \%$ dos empregos totais gerados na silvicultura e na exploração florestal do Brasil, contabilizando um total de quase 7 mil pessoas empregadas. A indústria do desdobramento e a da fabricação de produtos de madeira no Paraná participaram, respectivamente, com 18 e $24 \%$ do total de empregos nacionais gerados.

Apesar da importância do setor florestal para a economia paranaense, alguns analistas indicam que o setor pode ser bastante prejudicado na próxima década, em vista da possibilidade de uma escassez de madeira, fenômeno denominado popularmente com "apagão florestal". As bases para essas pressuposições vieram a partir de análises realizadas por Bacha (2000) e STCP (2004), que focaram um desequilíbrio entre a necessidade e disponibilidade de madeira no Brasil. 
O "apagão florestal", em geral, tem sido levantado como uma bandeira para requerer ações efetivas do governo em apoio ao setor produtivo florestal, uma vez que as ações então desenvolvidas teriam levado a uma diminuição da oferta de madeira. Segundo STCP (2005), a "síndrome do apagão" foi importante para trazer de volta, no âmbito do governo, a discussão econômica relacionada às florestas, até então muito concentrada em aspectos ambientais.

No período de 1999 a 2005, foi evidenciado um forte aumento no preço da madeira, o que, de fato, corrobora a tese de sua escassez no período considerado. Conforme o Instituto Brasileiro de Geografia e Estatística (IBGE, 2007) e a Secretaria de Agricultura e Abastecimento do Paraná (SEAB, 2006), o preço da madeira em tora entre 1999 e 2005 praticamente dobrou.

Uma questão relevante a saber é se esse aumento do preço é decorrente de uma queda na oferta ou de um aumento na demanda de madeira. Em outras palavras, saber se o "apagão florestal" se deve, fundamentalmente, à influência de fatores relacionados à produção de madeira (oferta) ou ao seu consumo (demanda).

O objetivo deste trabalho é analisar se o aumento de preço da madeira em tora proveniente de silvicultura no Paraná entre 1999 e 2005 foi provocado, principalmente, por fatores relacionados à demanda ou à oferta de madeira.

\section{MATERIAL E MÉTODOS}

\section{Material}

Este trabalho foi realizado a partir de dados secundários gerados pelo Instituto Brasileiro de Geografia e Estatística (IBGE, 2007), os quais foram agregados para o Paraná e dizem respeito à produção total de madeira em tora proveniente da silvicultura.

Foram coletadas as séries referentes ao valor da produção e quantidade produzida de madeira em tora no período entre 1999 e 2005 . A escolha desse período para análise deve-se ao forte aumento do preço da madeira nessa época.

A evolução do preço foi obtida através da razão entre o valor da produção e a quantidade produzida de madeira em tora de silvicultura para o Paraná (equação 1).

$$
P=\frac{V P}{Q P}
$$

Em que: $\mathrm{P}=$ preço $\left(\mathrm{R} \$ / \mathrm{m}^{3}\right)$;

$\mathrm{VP}=$ valor da produção $(\mathrm{R} \$)$;

$\mathrm{QP}=$ quantidade produzida $\left(\mathrm{m}^{3}\right)$.

A série de preço foi corrigida pelo Índice de Preço ao Consumidor Amplo (IPCA), base de dezembro de 2005, índice este publicado pelo IBGE (2007). Os dados utilizados foram de acordo com a tabela 1 .

Tabela 1. Dados referente ao mercado de madeira em tora de silvicultura do estado do Paraná.

Table 1. Data regarding to round wood market from the state of Paraná.

\begin{tabular}{lccc}
\hline Ano & Quantidade $\left(\mathbf{m}^{\mathbf{3}}\right)$ & Preço $\left(\mathbf{R} \mathbf{\$} / \mathbf{m}^{\mathbf{3}}\right)$ & Tendência \\
\hline 1999 & 12.505 .715 & 33,00 & 1 \\
2000 & 12.269 .070 & 32,49 & 2 \\
2001 & 13.501 .571 & 25,79 & 3 \\
2002 & 12.505 .377 & 41,10 & 4 \\
2003 & 20.088 .607 & 45,96 & 5 \\
2004 & 17.723 .676 & 60,90 & 6 \\
2005 & 22.835 .828 & 63,63 & 7 \\
\hline
\end{tabular}

\section{Métodos}

A metodologia empregada neste estudo consistiu basicamente de duas etapas. Primeiro foram estimados modelos de tendência, os quais calcularam as taxas de crescimento anual do preço e da quantidade produzida de madeira em tora para o Paraná no período considerado. Em seguida, foram 
relacionados os resultados de acordo com as possibilidades de deslocamento das curvas de oferta e demanda (ALMEIDA, 2006).

Foi assumido que o mercado de madeira em tora para o estado opera em uma estrutura com relativa concorrência, que as elasticidades do preço da demanda e da oferta não são completamente elásticas ou inelásticas (inclinação vertical ou horizontal) e que os agentes de mercado agem de forma racional, pressuposições estas que não se distanciam da realidade do setor.

Estimativa da taxa de crescimento anual composta

As estimativas das taxas de crescimento anuais para o preço e a quantidade produzida de madeira em tora proveniente de silvicultura do Paraná foram obtidas conforme Gujarati (2000).

De acordo com Gujarati (2000), o processo de cálculo da taxa de crescimento pode ser realizado da maneira descrita a seguir.

Seja Yt = QtMT (produção da madeira em tora no instante t) ou PtMT (preço da madeira em tora no instante t) conforme a equação (2):

$$
Y_{t}=Y_{0}(1+r)^{T}
$$

Em que: $Y_{t}=$ produção ou preço considerando a taxa $r$ ao longo do tempo $T$;

$Y_{0}=$ produção ou preço inicial;

$r=$ taxa composta;

$T=$ período;

Calculando o logaritmo natural, pode-se reescrever a equação (2) conforme (3):

$$
\ln Y_{t}=\ln Y_{0}+T \ln (1+r)
$$

Sejam $\beta_{1}=\ln Y_{0}$ e $\beta_{2}=\ln (1+r)$ e adicionando o termo de perturbação $\varepsilon_{t}$ na equação (3), obtêm-se a equação (modelo) (4):

$$
\ln Y_{t}=\beta_{1}+\beta_{2} T+\varepsilon_{t}
$$

Conforme o modelo (4), o coeficiente de inclinação ( $\beta 2)$ mede a variação proporcional constante em Y para uma dada variação absoluta no valor do regressor, nesse caso, a variável T. A taxa de crescimento percentual em Y no decurso do período de 1999 a 2005 é obtida através do cálculo do antilog de $\beta 2$, com uma posterior subtração por 1 e, por fim, a multiplicação do resultado por 100.

Para uma melhor visualização da taxa de crescimento da produção e preço de madeira, foram apresentadas graficamente suas evoluções e respectivas tendências lineares. A representação da tendência linear das séries foi obtida através do software Microsoft Excel.

Estimação e avaliação dos modelos

Os modelos de tendência foram estimados pelo método dos mínimos quadrados ordinários (MQO). Para verificação da qualidade do ajuste, adotou-se a estatística t de Student, considerando significativos valores em 5\% de probabilidade pelo teste bicaudal (GUJARATI, 2000; HILL et al., 1999).

Relações entre as taxas de crescimento calculadas e os deslocamentos das curvas de oferta e demanda

A partir dos sinais das taxas de crescimento calculadas, foi determinado se o comportamento do mercado de madeira em tora no Paraná foi explicado, principalmente, por fatores ligados à sua curva de oferta ou de demanda. A base teórica para esse julgamento foi o modelo desenvolvido por Marshall (1890), o qual permanece inalterado até os dias de hoje.

A obtenção de uma taxa de crescimento positiva para o preço $(\mathrm{p}+)$ e quantidade $(\mathrm{q}+)$ sugere um deslocamento da demanda para direita (1) maior do que qualquer deslocamento da oferta, seja este para esquerda (A) ou direita (B). Caso as taxas de crescimento encontradas sejam negativas para o preço (p-) e quantidade (q-), o deslocamento mais influente é da demanda para esquerda (2) (Figura 1).

Ao contrário da demanda, caso o deslocamento mais importante seja da oferta, inevitavelmente é obtida uma direção inversa dos sentidos da quantidade e do preço (p- e q+ ou p+ e q-). Um aumento da oferta para a direita (3) maior que qualquer deslocamento da demanda, seja este para esquerda ou direita (A ou B), implica um aumento da quantidade produzida (QP menor que QP1, QP2 e QP3) e uma queda do preço (P maior que P1, P2 e P3). No caso de um deslocamento dominante da oferta para a esquerda (4)), é obtido um aumento do preço (P menor que P1, P2 e P3) e queda na quantidade (QP maior que QP1, QP2 e QP3) (Figura 2). 


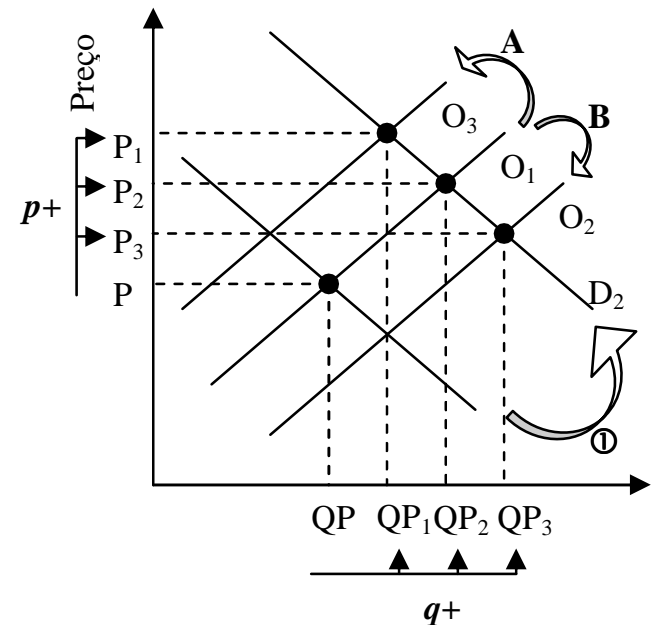

Quantidade Produzida

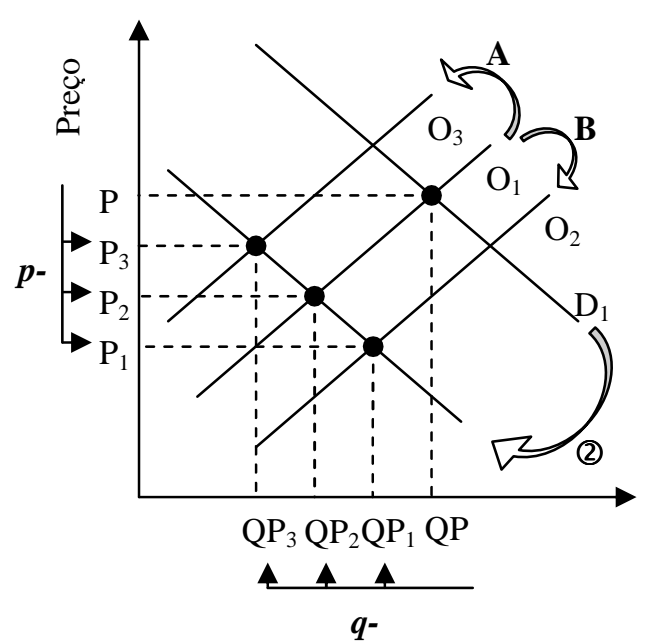

Quantidade Produzida

Figura 1. Comportamento do mercado considerando um deslocamento dominante da demanda para direita e esquerda.

Figure 1. Market behavior considering a dominant displacement of the demand to right and to the left.

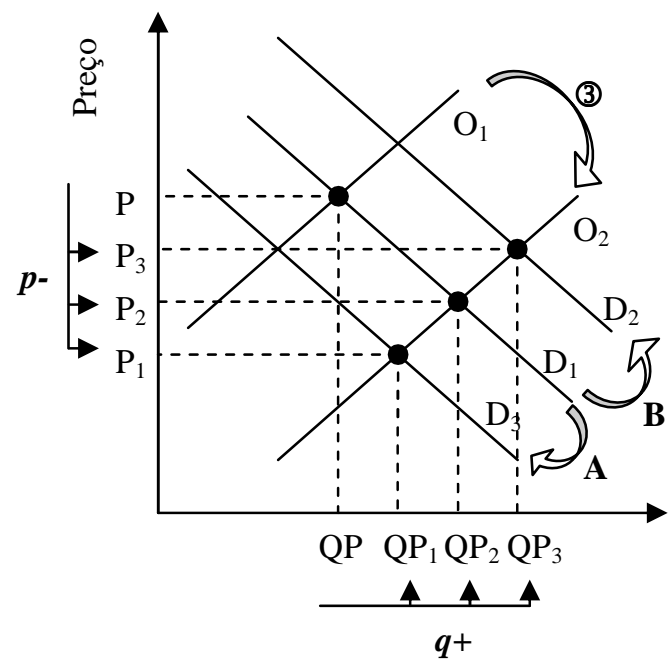

Quantidade Produzida

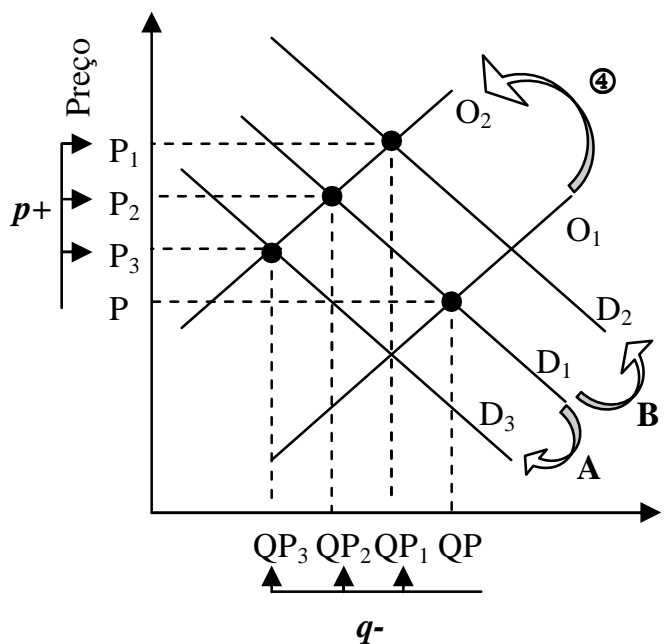

Quantidade Produzida

Figura 2 Comportamento do mercado considerando um deslocamento dominante da oferta para direita e esquerda.

Figure 2. Market behavior considering a dominant displacement of the supply to the right and to the left.

\section{RESULTADOS E DISCUSSÕES}

Conforme as equações 5 e 6 , a produção e o preço da madeira em tora proveniente de silvicultura no Paraná, no período de 1999 a 2005, aumentaram, respectivamente, a uma taxa de $10 \%$ e $14 \%$ ao ano. Todos os coeficientes calculados foram significativos com $1 \%$ de probabilidade de erro.

$$
\ln \hat{Q}_{t}^{M T}=16,13+0,10 T_{t}
$$


Teste $t(140,28)(4,08)$

$\mathrm{R}^{2}=0,78$

$\ln \hat{P}_{t}^{M T}=3,18+0,14 T_{t}$

Teste $t(20,88)(4)$

$\mathrm{R}^{2}=0,76$

Para facilitar a visualização dos resultados, eles são apresentados na figura 3.

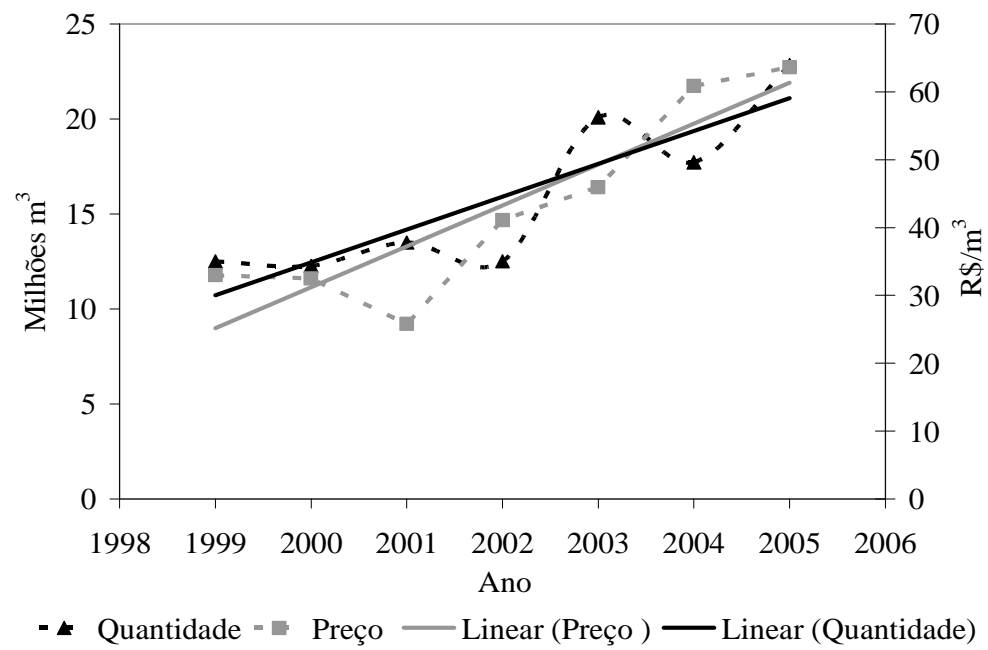

Figura 3. Evolução do preço e da quantidade produzida de madeira em tora paranaense e suas respectivas tendências lineares de crescimento entre 1999 e 2005.

Figure 3. Evolution of round wood price and round wood quantity from Paraná and their respective linear growth tendencies between 1999 and 2005.

Conforme as taxas de crescimento calculadas e as tendências lineares representadas pela figura 1 , nota-se que o aumento do preço da madeira em tora no período analisado foi acompanhado por um aumento da quantidade produzida. Essa situação reflete um deslocamento dominante da demanda para a direita, ou, em outras palavras, independentemente do movimento da curva de oferta (direita ou esquerda), a determinação do comportamento do mercado se deu via deslocamento da demanda.

Os resultados encontrados sugerem que o fator determinante para o crescente aumento do preço da madeira no período de 1999 a 2005 deveu-se a variáveis ligadas à demanda de madeira em tora. Dessa forma, fatores ligados ao consumo de bens, como, por exemplo, renda, preço de bens relacionados, gostos e preferência, foram muito mais importantes do que aqueles ligados à oferta de madeira.

O entendimento dos resultados encontrados, bem como a preocupação em torno de uma provável escassez de madeira, depende do conhecimento da história do setor florestal paranaense, pelo menos dos últimos 20 anos.

A partir de 1986, depois de 20 anos de incentivos fiscais, a atividade florestal deixou de contar com créditos diretos para o setor (SANTANA, 1999). Somando-se a isso, a partir de meados dos anos 80 foi crescente a regulação ambiental e a complexidade legal para implantação e exploração de florestas produtivas.

Entretanto esses fatores não foram determinantes e nem os principais responsáveis pela escassez de madeira e pelo aumento do seu preço nos últimos anos. Os resultados do trabalho indicam que o impacto do fim dos incentivos fiscais na área de plantações florestais não foi tão significativo. Nesse aspecto, ressaltam-se as empresas de papel e celulose, que foram as empresas que melhor souberam aproveitar a política de incentivos fiscais. Com o fim desses incentivos, essas empresas continuaram a manter padrões de reflorestamento suficientes para suprirem suas necessidades, haja vista o alto custo de capital inerente a esse setor.

De acordo com a Associação Brasileira de Celulose e Papel (BRACELPA, 2005), entre 1980 e 1988, o setor de papel e celulose no Paraná detinha um total de área reflorestada num patamar de 150 a 
200 mil hectares, passando para um patamar entre 250 e 300 mil hectares após o fim dos incentivos que perduraram até o ano de 2005.

Outro fator importante a ser considerado deve-se à grande evolução da produtividade ocorrida na década de 80, a qual minimizou a necessidade de aumento nas áreas plantadas para suprir as necessidades da indústria. Segundo a Empresa de Pesquisa Agropecuária e Extensão Rural de Santa Catarina (EPAGRI, 2005), enquanto que a produtividade média das florestas de pinus e eucaliptus, nos anos oitenta, era de cerca de 23 e $27 \mathrm{~m}^{3} / \mathrm{ha} / \mathrm{ano}$, respectivamente, atualmente se aproxima de $40 \mathrm{~m}^{3} / \mathrm{ha} / \mathrm{ano}$.

Por fim, não pode ser ignorado o fato de que, com o fim dos incentivos fiscais, a indústria de papel e celulose, maior detentora de florestas no Paraná, não ficou totalmente desamparada de apoios governamentais. Em 1987 foi lançado o II Programa Nacional de Papel e Celulose (PNPC), o qual contabilizou um investimento superior a US\$ 6 bilhões no período de 1987 a 1995. Em 1981, o setor de papel e celulose participava com 1,92\% no total das operações de financiamentos aprovadas pelo Banco Nacional de Desenvolvimento Econômico e Social (BNDES), e em 1990 essa participação chegou a $16,3 \%$ do total de financiamentos concedidos pelo banco. Poucos setores da economia brasileira foram tão beneficiados pelo governo quanto a indústria de papel e celulose (SANTANA, 1999).

Em relação à demanda de madeira, esta foi determinada principalmente pelo mercado externo, visto o fraco desempenho da economia interna no período analisado. A renda nacional brasileira cresceu apenas $3,76 \%$ ao ano no período analisado, valor este próximo ao apresentado pelas economias já desenvolvidas e inferior ao de países em desenvolvimento e com situações semelhantes (INSTITUTO DE PESQUISA ECONÔMICA APLICADA - IPEA, 2007).

Em relação ao desempenho das exportações brasileiras, destaca-se a indústria de compensado, a qual teve uma queda de 3,5\% no consumo interno entre 1999 e 2005 e um crescimento de $17 \%$ no seu volume exportado no mesmo período. O consumo interno da indústria de serrado de coníferas e papel cresceram, respectivamente, $4,2 \%$ e 17,2\% ao ano. Já as suas exportações aumentaram a uma taxa de 5,5\% e 21,74\% ao ano (FOOD AND AGRICULTURE ORGANIZATION OF THE UNITED NATIONS - FAO, 2007).

Especificamente para o Paraná, objeto da pesquisa, foi observado um aumento, respectivamente, de $86 \%, 174 \%$ e $116 \%$ nas exportações de serrados, compensados e papel e celulose entre os anos de 1999 e 2005 (SECRETARIA DE COMÉRCIO EXTERIOR - SECEX, 2007).

Dentre os principais fatores que contribuíram para o incremento das exportações de bens de madeira e consequente demanda interna de madeira em tora podem ser destacados a desvalorização cambial no período, a lei Kandir, o bom momento da economia mundial e a crescente abertura da economia brasileira, entre outros.

Entre 1998 e 2005, a taxa de câmbio (R\$/US\$) desvalorizou 110\%, saindo de um dólar médio em 1998 de $\mathrm{R} \$ 1,16$ para $\mathrm{R} \$ 2,43$ em 2005. O crescimento médio da renda mundial entre 1999 e 2005 foi de 4,04\%, levemente superior à média histórica (1961-2005) de 3,86\% (IPEA, 2007). A lei Kandir, a partir de 1996, também foi um incentivo às exportações, na medida em que desonerou os exportadores do pagamento do ICMS Estadual.

\section{CONCLUSÕES}

- A análise indica que o aumento do preço da madeira entre 1999 e 2005 foi devido, fundamentalmente, a variáveis relacionadas à demanda externa de madeira. Sendo assim, inferir que esse aumento é derivado de uma maior regulação ambiental ou de uma redução de apoio governamental, ou mesmo de redução da área plantada é, no mínimo, uma constatação incompleta. Deve-se, portanto, inserir as variáveis de demanda para um melhor entendimento do desequilíbrio existente na época e de futuros desequilíbrios.

- O sucesso de qualquer política que afete o mercado de madeira em tora, como, por exemplo, o incentivo à entrada de pequenos produtores de floresta, depende fundamentalmente de uma boa análise e entendimento da dinâmica desse mercado.

\section{REFERÊNCIAS}

ALMEIDA, A. N. Estudo econométrico da demanda e oferta de madeira em tora para o processamento mecânico no estado do Paraná. 217 f. Dissertação (Mestrado em Engenharia Florestal) - Centro de Ciências Florestais e da Madeira, Universidade Federal do Paraná, Curitiba. 2006.

ANDRETTA, G. C. Valor bruto da produção agropecuária paranaense 1997 e 2004. Curitiba: SEAB/DERAL, v. 89, 2006, p. 89. 
ASSOCIAÇÃO BRASILEIRA DA INDÚSTRIA DE MADEIRA PROCESSADA MECANICAMENTE. Setor de processamento mecânico da madeira no Estado do Paraná. Disponível em: <http://www.abimci.com.br/port/06Docs/06Quadro Docs.html>. Acesso em: 02/08/2007.

ASSOCIAÇÃO BRASILEIRA DE CELULOSE E PAPEL (BRACELPA). Anuário Estatístico 2005/2006. São Paulo, 2006.

BACHA, C. J. C. Evolução da produção brasileira de Madeira: Haverá Escassez? Preços Agrícolas, Piracicaba, p. 13 - 16, jul. 2000.

BRASIL. Ministério do Desenvolvimento Indústria e do Comércio Exterior. Secretaria de Comércio Exterior (SECEX). Disponível em: <http://aliceweb.desenvolvimento.gov.br/>. Acesso em: 30/07/2007.

EMPRESA DE PESQUISA AGROPECUÁRIA E EXTENSÃO RURAL DE SANTA CATARINA (EPAGRI). O Setor Florestal de Santa Catarina. Florianópolis, 2005. 1 CD-ROM.

FOOD AND AGRICULTURE ORGANIZATION OF THE UNITED NATIONS (FAO). Disponível em: <http://faostat.fao.org/site/381/default.aspx>. Acesso em: 10/08/2007.

GUJARATI, D. N. Econometria básica. 3. ed., São Paulo: Makron Books, 2000. 846 p.

HILL, C.; GRIFFITHS, W.; JUDGE, G. Econometria. São Paulo: Saraiva, 1999. 408 p.

INSTITUTO BRASILEIRO DE GEOGRAFIA E ESTATÍSTICA (IBGE). Anuário Estatístico Brasileiro. Brasília, DF: Diretoria de Pesquisas, Departamento de Agropecuária, Pesquisa Produção Vegetal e da Silvicultura. Várias edições.

INSTITUTO DE PESQUISA ECONÔMICA APLICADA (IPEA). Disponível em: <http://www.ipeadata.gov.br/ipeaweb .dll/ipeadata?1357421>. Acesso em: 02/08/2007.

MARSHALL, A. Princípios de economia: tratado introdutório. Tradução revista: Rômulo de Almeida e Ottolmy Strauch. 3. ed. São Paulo: Nova Cultural, 1998. v. 2.

PARANÁ. SECRETARIA DE AGRICULTURA E ABASTECIMENTO. Relatório de Atividades 2006. Curitiba, 2006.

SANTANA, L. V. Avaliação de políticas públicas setoriais: o fator retroalimentador na demanda de políticas econômicas pelo setor florestal no Brasil. o complexo papel-celulose.. $499 \mathrm{f}$. Tese (Doutorado em Ciências Florestais) - Setor de Ciências Agrárias, Universidade Federal do Paraná, Curitiba, 1999.

STCP Engenharia de Projetos Ltda. O desequilíbrio do mercado de tora de pinus no Brasil. STCP Informativo, Curitiba, n. 7, 2004.

. O apagão e o Brasil Florestal em 2020. STCP Informativo, Curitiba, n. 8, 2005. 